\title{
ANTIOXIDANT ACTIVITY AND BIOGENIC SYNTHESIS OF SELENIUM NANOPARTICLES USING THE LEAF EXTRACT OF ALOE VERA
}

\author{
JAY VYAS a, SHAFKAT RANA ${ }^{b}$ \\ aFaculty of Science, Pacific Academy of Higher Education and Research University, Udaipur (Rajasthan) India 313001, bP. G. Department of \\ Botany, Shri Govind Guru Govt. College, Banswara (Rajasthan) India 327001 \\ Email: Jay88vyas@gmail.com \\ Received: 15 Feb 2017, Revised and Accepted: 10 May 2017
}

\begin{abstract}
Objective: The objective of this present study were to the biogenic synthesis of selenium nanoparticles using Aloe vera extract and check it's antioxidant potential by ABTS, DPPH and FRAP assays.

Methods: In this study we investigated the clove of Aloe vera, which is used for the synthesis of selenium nanoparticles were characterized by using UV-Visible (UV-VIS) spectrophotometer, Transmission electron microscopy (TEM), Fourier transform spectroscopy (FTIR) and Energy dispersive XRay spectroscopy (EDAX) and ABTS, DPPH and FRAP assays for checked it's antioxidant potential.

Results: The present study was carried out to synthesis of Selenium nanoparticles using extract of Aloe vera. UV-Vis Spectra at 350 nm with Aloe vera extract and observed as hollow and spherical particles in size ranging 7-48 $\mathrm{nm}$ which is found more stable more than two months. EDAX analysis was carried out to check the presoak of Selenium in nanoparticles. Results of EDAX, confirmed its present. TEM and SEAD represented addition evidence of formation of nanoparticles whereas SEAD indicates the particles were crystalline in nature. FT-IR analysis was carried out to identify the possible bio molecules and Aloe vera extract-metal ions interaction responsible for formation and stabilization of selenium nanoparticles. FRAP, ABTS and DPPH assay results sequester that Selenium nanoparticles prepared using Aloe vera extract possess more activity than extract alone.
\end{abstract}

Conclusion: The bio molecules of Aloe vera extract acted as stabilizing as well as capping agent leading to the formation of Selenium nanoparticles. Selenite has been proven to have antioxidant activity and is being used as chemoprevention agent in cancer diagnosis but same time it is toxic also. Elemental Selenium i.e. Selenium nanoparticles are less toxic form of selenium.

Keywords: Biogenic synthesis, Selenium nanoparticles, Aloe vera, Antioxidant potential

(C) 2017 The Authors. Published by Innovare Academic Sciences Pvt Ltd. This is an open access article under the CC BY license (http://creativecommons.org/licenses/by/4.0/) DOI: http://dx.doi.org/10.22159/ijcpr.2017v9i4.20981

\section{INTRODUCTION}

Since the last decade, Nanotechnology is the active area of research. Nanotechnology is emerged as an interdisciplinary approach in biochemical application with focus on the cure of different disease like Alzimer, cancer etc [1].

Production of nanoparticles can be achieved through chemical and physical method [2, 3]. In bio nanotechnology the source of synthesis like microorganisms, enzyme, plants and plant extracts as possible methods to avoid the use of toxic chemical and expensive physical tools. The using of plant materials is an economical, ecofriendly and reliable technique [4].

In nanotechnology, nanoparticles defined as small object that behaves as a whole unit in terms of its transport and having one more dimensions of the order of $100 \mathrm{~nm}$ or less [5]. Nanoparticles shows size and shape dependent properties which are interest for application ranging from bio sensing and catalyst to optics, antimicrobial activity, antioxidant activity, computer transistors, electrometers, chemical sensors and wireless electronic logic and memory scheme [6].

Selenium(Se), belonging to group 16 of the periodic table having atomic no. 34 and atomic mass 78.96 amu. It is widely used for solar cells, rectifiers, photographic exposure meter and xerography due to its photoelectric and semiconductor properties. Selenium occurs in variety of oxidation state like selenate $\left({ }^{\mathrm{SeO}_{4}^{2-}}\right)$, selenite $\left({ }^{\mathrm{S}_{\mathrm{B}}^{2-}}\right)$, where in oxidation states +6 and +4 [4].

Selenium having great potential and properties in the field of medicine, physics, biology and chemistry. The selenium nanoparticles are also used as antioxidants, antimicrobial, anticancer agent but it's highly toxic so preparation of stable selenium nanoparticles as nontoxic biomedical application is still challenge [7].

Selenium nanoparticles has been synthesized by different biological source like Bacillus sp. MshI [8], Klebsiella pneumonia [9], Bougainvillea spectabilis [10], leaves of lemon [11], resin extract of grapes [12].

The fruits and vegetables are rich in antioxidants including polyphenolic compounds; vitamin $\mathrm{E}$ and vitamin $\mathrm{C}$ are believed to be the effective nutrients in the preventions of the oxidative stress related diseases [13].

The oxidative stress in occurs due to the reactive oxygen species(ROS) which is generated by redox process in metabolism these species are highly reactive and harmful to the cells the antioxidants enzyme and non enzymatic compounds for complete scavenging of ROS. A study also shows that prevention of free radicals induced disease by the antioxidant substances [14].

Aloe vera grows wild in tropical climates around the world and is cultivated for agriculture and medicinal uses. It is also used for decorative purposes and grows successfully indoors as a potted plant. Aloe vera leaf contains many vitamins mostly vitamin A, C and F. It also contains free anthraquinones and their derivatives Barbaloin, Anthron-c-glycosides and chromones. Aloe vera having antitumor, antioxidant, anti inflammatory, antimicrobial and anti fungal activities [15].

Therefore, the present study in aimed to evaluate the reducing potential of Aloe vera leaf in the synthesis and stabilization of selenium nanoparticles and compare the efficiency of ABTS, DPPH and FRAP assay to estimate antioxidant activity of Aloe vera extract and selenium nanoparticles synthesized by Aloe vera extract. 


\section{MATERIALS AND METHODS}

\section{Preparation of Aloe vera extract}

A $30 \mathrm{gm}$ portion of thoroughly washed Aloe vera leaves. Finely cut into $20 \mathrm{ml}$ of sterile distilled water. The extract was then filtered through Whatman filter paper no. 1 and stored at $4{ }^{\circ} \mathrm{C}$ for further work.

\section{Synthesis of metal nanoparticles}

Flask containing $25 \mathrm{ml} 5 \mathrm{mmol} \mathrm{Na}_{2} \mathrm{SeO}_{3}$ solutions was kept on magnetic stirrer. Then drop wise addition of Aloe vera extract was made in flask containing $\mathrm{Na} 2 \mathrm{SeO} 3$ solution until color of sodium selenite solution changed. From this solution $5 \mathrm{ml}$ was taken which was used as a control. Remaining $20 \mathrm{ml}$ solution was kept in shaker in dark for $72 \mathrm{~h}$. After few days the color change of the solution was observed [11].

\section{UV-Vis spectra analysis}

The reduction of metallic selenium ions was observed by measuring the UV-Vis spectrum after 10 to $15 \mathrm{~min}$ of color change. A small aliquot was drawn from the solution and a wavelength from $250 \mathrm{~nm}$ to $700 \mathrm{~nm}$ on UV-Vis spectrophotometer (Optizon Double beam 3220) [10].

\section{TEM analysis}

Transmission Electron Microscopic (TEM) analysis was performed with Techni 20 (Philips, Holland). A thin film of the sample was prepared on a carbon coated copper grid by dropping a very small amount of the sample on the grid. The Aloe vera extract containing Se nanoparticles were subjected to centrifugation at $13000 \mathrm{rpm}$ for $10 \mathrm{~min}$. The pellet thus recovered was subjected to washing by its re-suspension in deionized water followed by centrifugation at $13000 \mathrm{rpm}$ for $10 \mathrm{~min}$, to remove possible organic contamination present in nanoparticles. Finally, pellet was freeze dried using a lyophilizer (Labconco, Kanas, USA) [11].

\section{EDAX analysis}

EDAX analysis was carried out on EDAX XL-30 operating at 15$25 \mathrm{KeV}$. Incorporation of selenium nanoparticles in gauze cloth. Nanoparticles suspension was poured on the gauze cloth discs (diameter $1 \mathrm{~cm}$ ) and there discs were dried at $36^{\circ} \mathrm{c}$ for $7 \mathrm{~d} \mathrm{[11].}$

\section{Sample preparation for Fourier transform spectroscopy (FTIR)}

Metal containing Aloe vera extract for Fourier Transform Infrared (FTIR) analysis was prepared by mixing $5 \mathrm{mg}$ metal salt in $10 \mathrm{ml}$ plant extract. This metal containing plant extract was incubated at room temperature for $1 \mathrm{~h}$. After $1 \mathrm{~h}$ incubation, this metal containing leaf extract was dried in Petri plate. After drying, particles were scraped using blade. So, powder of synthesized nanoparticles was obtained. Then spectral scan analysis was carried out at wave number ranging from 400-4000 cm-1 by using a FT-IR spectrometer (Perkin Elmer, Spectrum GX) with resolution of $0.15 \mathrm{~cm}-1$ to evaluate functional groups that might be involved in sorption process [11].

\section{Antioxidant assay}

\section{ABTS assay}

For ABTS assay, the procedure followed the method of [16] with some modifications. The percent inhibition of ABTS radical by plant extracts were determined by the ability of plant extracts to scavenge the cationic free radical ABTS. Different concentrations (100 to 600 $\mu \mathrm{g} / \mathrm{ml}$ ) of Aloe vera extract and the biogenic synthesized Se nanoparticles were separately mixed with $3 \mathrm{ml}$ of $0.1 \mathrm{mmol}$ ABTS and incubated in dark for $15 \mathrm{~min}$. The extent of decolorization was measured at $745 \mathrm{~nm}$. Rutin was used as standard and ABTS reagent without sample was used as control solution. The percent of scavenging inhibition capacity of ABTS+ of the extract was calculated from the following equation:

$$
\text { \%o inhibition }=\frac{(\text { Abs control }- \text { Abs sample })}{(\text { Abs control })} \times 100
$$

\section{DPPH assay}

The DPPH assay was done according to the method of [17] with some modifications. The radical scavenging and antioxidant potential of the plant extracts were determined by the ability of plant extracts to scavenge the stable free radical DPPH and convert it into Diphenyl picryl hydrazine. The degree of decolourization from purple to yellow color was measured spectrophotometrically at 517 $\mathrm{nm}$. Different concentrations (100 to $600 \mu \mathrm{g} / \mathrm{ml}$ ) of Aloe vera extract and the biogenic synthesized Se nanoparticles were separately mixed with $3 \mathrm{ml}$ of $0.1 \mathrm{mmol}$ DPPH and incubated in dark for $15 \mathrm{~min}$. Rutin was used as standard and DPPH methanol reagent without sample was used as control. The reaction mixture was mixed well and left in dark at room temperature for $30 \mathrm{~min}$. The absorbance was measured spectrophotometrically at $517 \mathrm{~nm}$. The scavenging ability of the plant extract was calculated using this equation:

$$
\text { DPPH Scavenging activity }(\%)=\frac{\text { (Abs control }- \text { Abs sample) }}{(\text { Abs control })} \times 100
$$

\section{FRAP assay}

The FRAP assay was done according to [18] with some modifications. This method is based on the reduction of ferric-tripyridyltriazine complex to its ferrous, coloured form in the presence of antioxidants. Readings of the coloured product (ferrous tripyridyltriazine complex) were then taken at $593 \mathrm{~nm}$. Different concentrations $(100-500 \mu \mathrm{g} / \mathrm{ml})$ of Aloe vera extract and the biogenic synthesized Se nanoparticles of $0.5 \mathrm{ml}$ was separately mixed with $2.5 \mathrm{ml}$ of FRAP reagent allowed to react at room temperature in the dark. Rutin was used positive control in this test. An increase in the absorbance with increasing concentration is directly proportional to the reducing power. Results are expressed in $\mu \mathrm{g}$ Ascorbic acid equivalent ( $\mu \mathrm{g}$ TE/mg de). BHT was used as reference.

\section{RESULTS AND DISCUSSION}

\section{Visual observation}

Reduction of metal salts into metal nanoparticles by the bio molecules is always accompanied by the color change of reaction medium. In the present study the colorless solution of sodium selenite is changed in light pink color after drop wise addition of Aloe vera leaf extract at zero h. As the reduction proceed, the color of reaction medium is gradually changed to dark pink color after $24 \mathrm{~h}$.

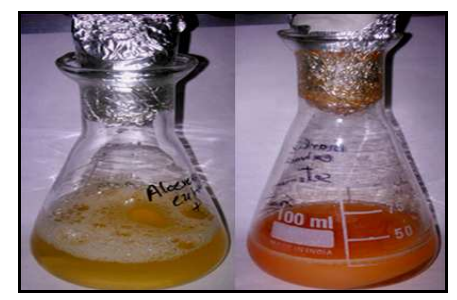

Fig. 1: Colour changed after reduction

\section{UV-visible spectroscopy}

In order to determine the formation of Selenium nanoparticles in the extract of Aloe vera, a spectral scanning procedure was carried out from $250 \mathrm{~nm}$ to $700 \mathrm{~nm}$. Colloidal solution exhibited absorption maxima at $350 \mathrm{~nm}$ (fig. 2).

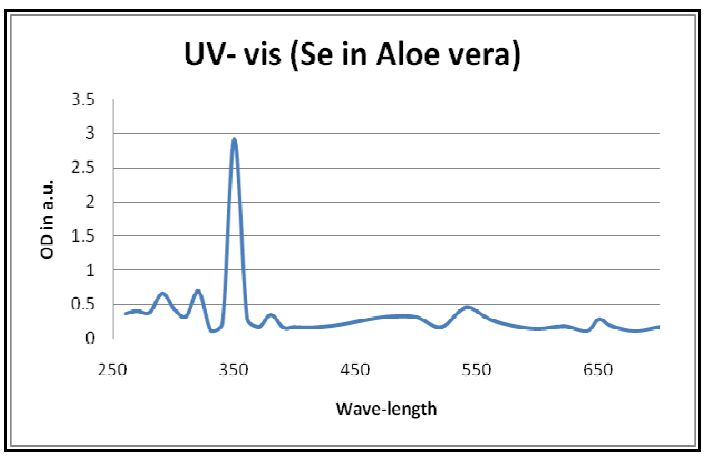

Fig. 2: UV-VIS spectra selenium nanoparticles 
Initially the colloidal solution appeared white in color but after incubation of a period of $24 \mathrm{~h}$, it turned to reddish brown in color. Building of absorbing maximum at $350 \mathrm{~nm}$ clearly indicates the gradual formation of particles during the incubation period.

\section{Transmission electron microscopy (TEM)}

TEM analysis of colloidal solution indicated the formation of selenium nanoparticles. (fig. 3) shows that size of particles, generated using Aloe vera extract ranges from 7-48 $\mathrm{nm}$. Formation of variable size of particles indicates that particles suggest that Aloe vera extract could form poly disperse nanoparticles. Fig. 3 shows Selected Area Electron Diffraction (SAED) of selenium nanoparticles.

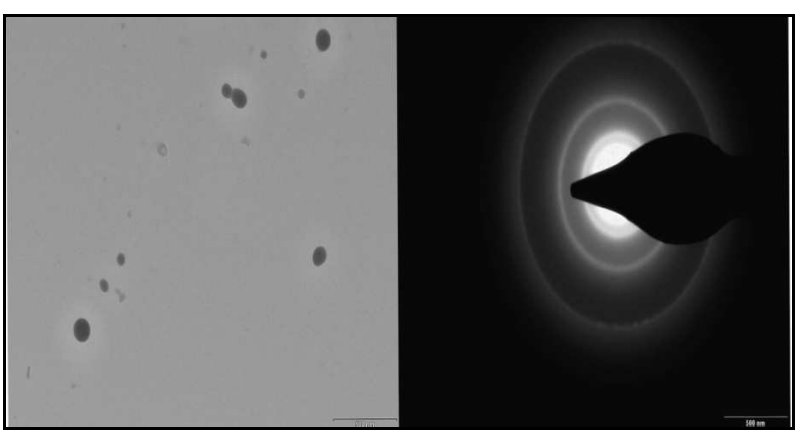

Fig. 3: TEM analysis of Selenium nanoparticles reveled size of particles 7-48 $\mathrm{nm}$

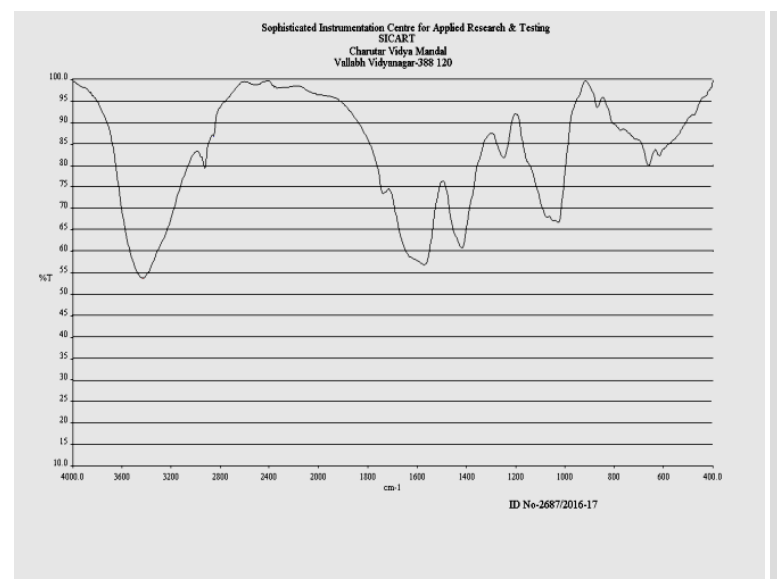

Fig. 4: FTIR spectrum of (A) Aloe vera extract and (B) Selenium nanoparticles synthesized by Aloe vera extract
Results shows that particles are crystalline in nature as diffraction ring appeared which correspond to diffraction angle of $(111,121$ and 311).

\section{Fourier transform infrared spectroscopy (FT-IR)}

FT-IR analysis was carried out to identify the possible bio molecules and plant extract-metal ions interaction responsible for formation and stabilization of silver nanoparticles. The result of FT-IR analysis of plant extract is presented in fig. 4 . The fig. 4 shows the spectrum of both the sample control (A) and test (B). Fig. 4 (B) shows the spectrum of the sample that contains selenium metal in Aloe vera extract or fig. 4 (A) shows the spectrum of the Aloe vera extract that did not contain metal selenium. Spectra A show the peaks at 3439.34, 2926.57, 2856.55, 1630.80, 1412.22, 1384.92, 1315.09, 1112.49, 779.36, 618.64 and $522.63 \mathrm{~cm}-1$. Similarly the transmission peaks of the sample (fig. $4 \mathrm{~B}$ ) that did not contain metal selenium were obtained at 3421.21, 2925.59, 2856.55, 1740.26, $1610.60,1415.00,1319.54,1250.14,1077.04,1033.46,812.77$, $775.97,675.86,599.98$ and $540.99 \mathrm{~cm}-1$.

Two absorption peaks located around 3400 and 4000 can be assigned as the absorption peak of $\mathrm{N}-\mathrm{H}$. The peaks located around 3000 and 3200 may be due to the presence of $\mathrm{C}-\mathrm{H}$ group. The absorption peaks around 2300 and 2000 can be assigned as the peaks of $\mathrm{CO}_{2}$. The absorption peaks around 1500 and 1800 can be assigned as the absorption peaks of $\mathrm{C}=\mathrm{O} / \mathrm{C}=\mathrm{N} / \mathrm{C}=\mathrm{C}$. The peaks around 1200 and 1100 were attributed to the stretching vibration of carboxyl group $(\mathrm{C}=0)$. The peaks around 1100 and 1000 may be due to the presence of $\mathrm{C}-\mathrm{O}$ group. Two absorption peaks around 600 and 500 may be due to the partial deuteration of amine or carboxyl group.

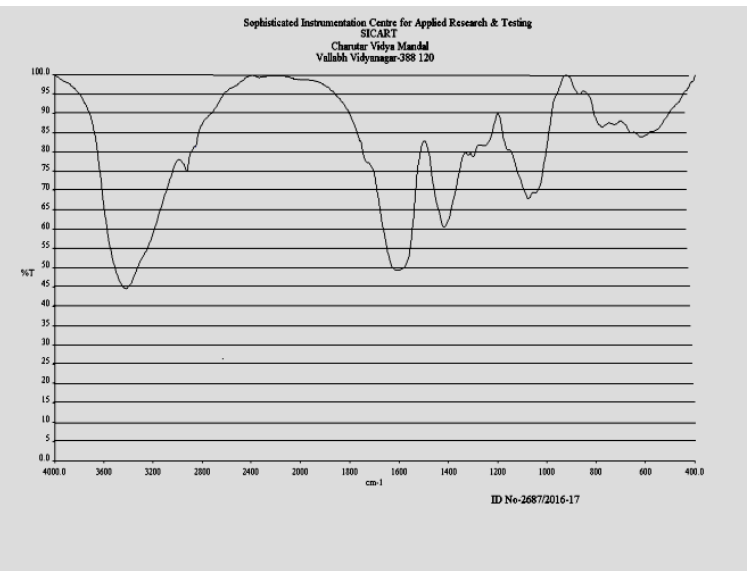

\section{Energy dispersive X-ray spectroscopy (EDAX)}

EDAX analysis gives qualitative as well as quantitative status of elements that may be involved in formation of nanoparticles. Fig. shows the elemental profile of synthesized nanoparticles using Aloe vera extract. The analysis revealed the highest proportion of Selenium (25\%) in nanoparticles followed by oxygen $(20 \%)$, sodium (10\%) P (10\%) S (8\%) etc.

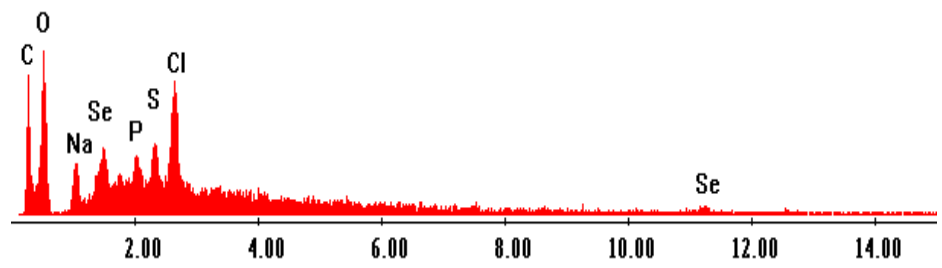

Fig. 5: EDAX spectrum of selenium nanoparticles

\section{Antioxidant assay}

\section{ABTS assay}

The reducing power of compounds is directly proportional to antioxidant activity of biogenic synthesized selenium nanoparticles was assessed by ABTS scavenging assay by using Rutin as a positive control. ABTS was a stable compound and accepts hydrogen or electrons from Aloe vera and synthesized Selenium nanoparticles. The results obtained in the ABTS assay showed effective free radical inhibition by both Aloe vera extract and synthesized Selenium 
nanoparticles. The average percentage inhibition of synthesized Selenium nanoparticles was $73 \%$ as compared to Aloe vera extract $54 \%$ at different concentrations used in this study and the activity increased with increasing concentrations. Fig. 6 indicates that synthesized Selenium nanoparticles containing Aloe vera extract that relatively strong ABTS radical scavenging activity.

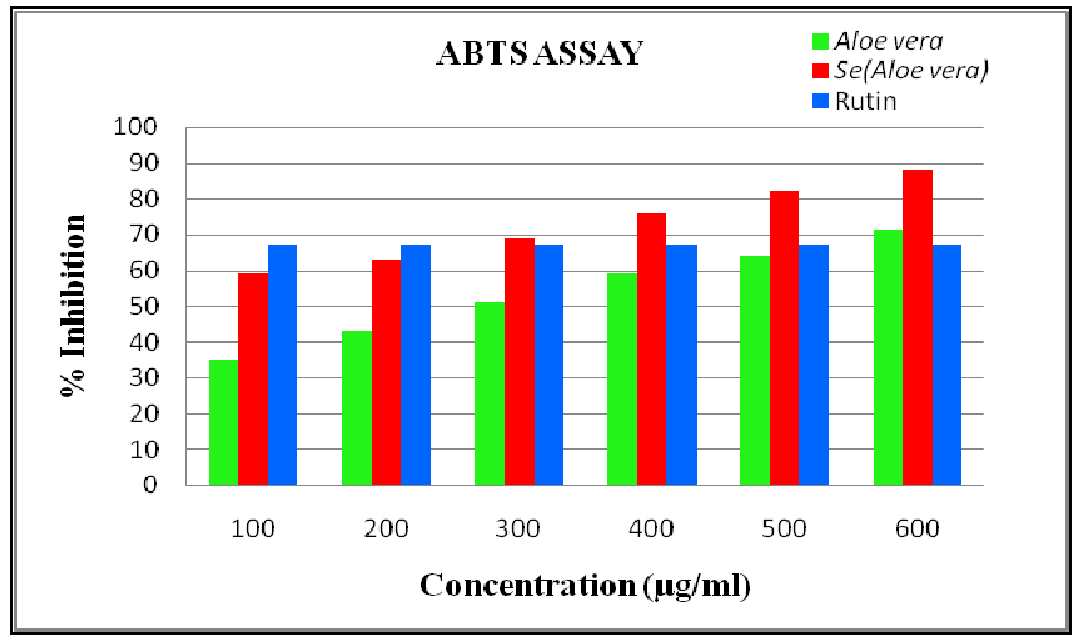

Fig. 6: ABTS assay showing enhanced antioxidant activity of synthesise selenium nanoaprticles

\section{DPPH assay}

Antioxidants are compounds that prevent the oxidation of essential biological macromolecules by inhibiting the propagation of the oxidizing chain reaction. The reducing power of compounds is directly proportional to antioxidant activity of biogenic synthesized selenium nanoparticles was assessed by DPPH scavenging assay by using Rutin as a positive control. DPPH was a stable compound and accepts hydrogen or electrons from Aloe vera and synthesized Selenium nanoparticles. The results obtained in the DPPH assay showed effective free radical inhibition by both Aloe vera extract and synthesized Selenium nanoparticles. The average percentage inhibition of synthesized Selenium nanoparticles was $67 \%$ as compared to Aloe vera extract $57 \%$ at different concentrations used in this study and the activity increased with increasing concentrations.

Fig. 7 indicates that synthesized Selenium nanoparticles containing Aloe vera extract that relatively strong DPPH radical scavenging activity. The present study was aimed to assess the antioxidant activity of Selenium nanoparticles in Aloe vera extract.

Keeping in mind the adverse effects of synthetic antioxidants, researchers have channeled their interest in preparing a new variety of natural antioxidants which are very effective to control the oxidative stress and hence prevent the initiation of disease propagation. The result of DPPH scavenging activity assay in this study indicates that the synthesize nanoparticles was potently active.

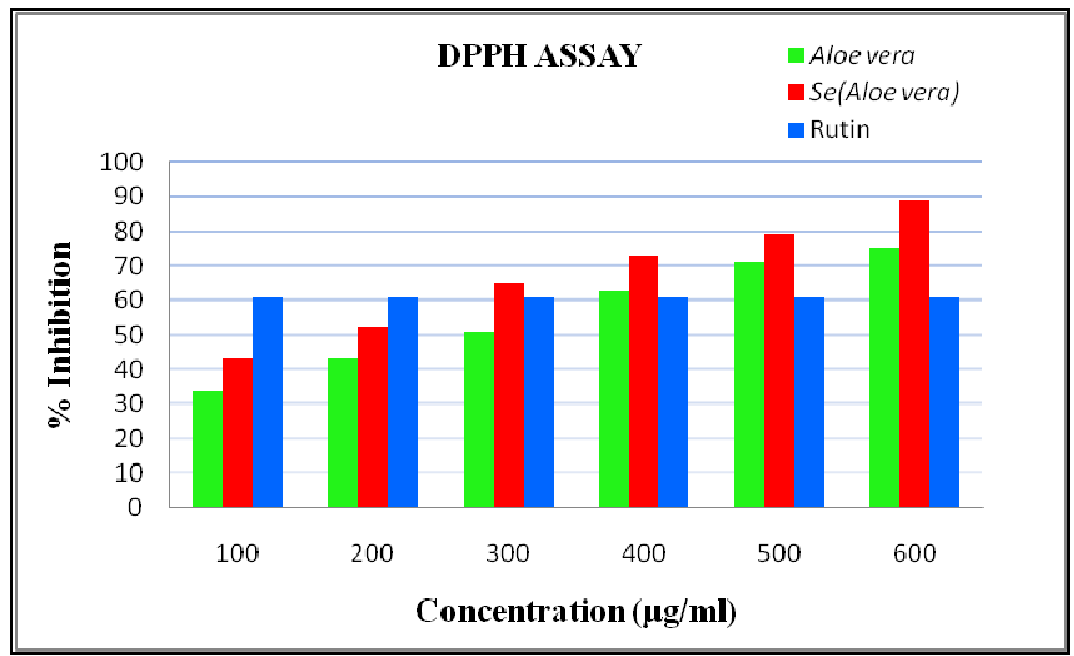

Fig. 7: ABTS assay showing enhanced antioxidant activity of synthesise selenium nanoaprticles

\section{FRAP assay}

In FRAP assay the change in absorbance is directly related to the combined or "total" reducing power of the electron donating antioxidants present in the reaction mixture of Selenium nanoparticles containing Aloe vera extract. According to FRAP assay Fig.8 shows reducing activity of biogenic synthesized Selenium nanoparticles and Aloe vera extract. Selenium nanoparticles showed more reducing activity than the Aloe vera extract and the reducing activity of Selenium nanoparticles was found with increasing concentrations. 


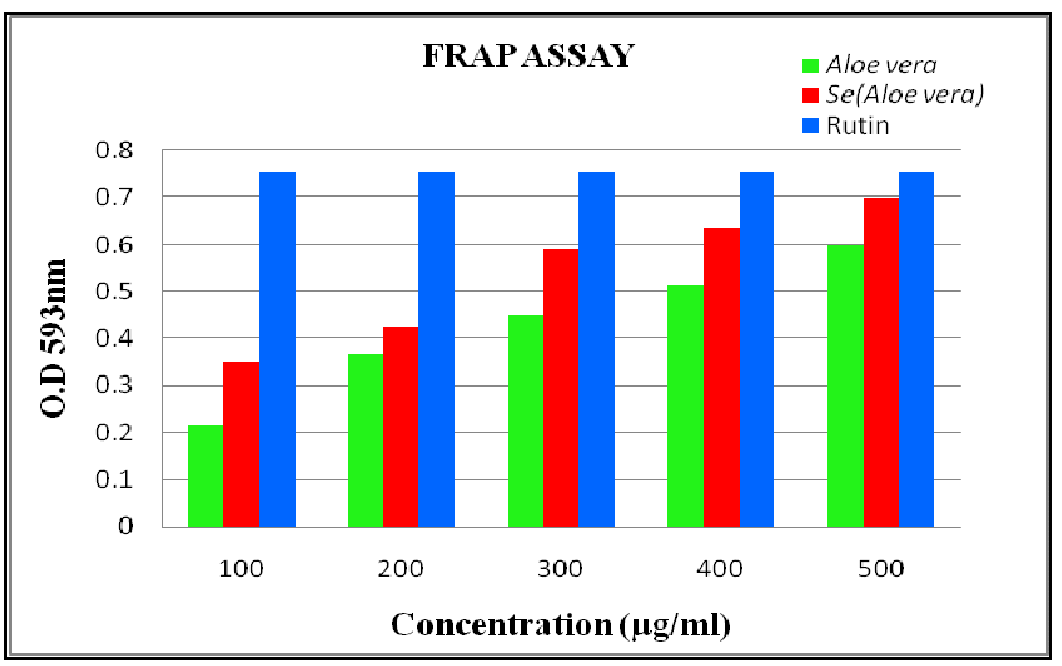

Fig. 8: FRAP assay showing enhanced antioxidant activity of synthesise Selenium nanoaprticles

\section{CONCLUSION}

The present study was carried out to synthesized Selenium nanoparticles using extract of Aloe vera. The bio molecules of Aloe vera extract acted as stabilizing as well as capping agent leading to the formation of Selenium nanoparticles. UV-Vis Spectra at $350 \mathrm{~nm}$ with Aloe vera extract and observed as hollow and spherical particles in size ranging 7-48 $\mathrm{nm}$ which is found more stable more than two months. EDAX analysis was carried out to check the presoak of Selenium in nanoparticles. Results of EDAX, confirmed its present. TEM and SEAD represented addition evidence of formation of nanoparticles whereas SEAD indicates the particles were crystalline in nature. Selenite has been proven to have antioxidant activity and is being used as chemoprevention agent in cancer diagnosis but same time it is toxic also. Elemental Selenium i.e. Selenium nanoparticles are less toxic form of selenium. FRAP, ABTS and DPPH assay results sequester that Selenium nanoparticles prepared using Aloe vera extract possess more activity than extract alone.

\section{ACKNOWLEDGMENT}

The authors are heartly grateful to Dr. Nilanjan Roy, Director, ARIBAS for his support and guidance. I am deeply thankful to Dr. C. L. Patel, CVM, for providing me with state of art facilities and infrastructure which was inevitable for my research work. My sincere thanks to Mr. Vikas and Mr. Sanjay of SICART for their promptness I would also like thank Jaideep Singh Rathore, Director, Wagrshree College, banswara (Rajasthan) for constantly motivating me.

\section{CONFLICT OF INTERESTS}

Declare none

\section{REFFERENCES}

1. P Gopinath, Sonit Kumar Gogoi, Pallab Sanpui, Anumita Paul, Arun Chatopadhyay, Siddharth Shankar Ghosh. Signaling gene cascade in silver nanoparticle induced apoptosis. Colloids Surf B 2010;77:240-5.

2. CB Murray, CR Kagan, MG Bawendi. Synthesis and characterization of monodisperse nanocrystals and close-packed nanocrystal assemblies. Annual Rev Mater Sci 2000;30:545-610.

3. P Ayyub, R Chandra, P Taneja, AK Sharma, R Pinto. Synthesis of nanocrystalline material by sputtering and laser ablation at low temperatures. Appl Phys 2001;73:67-73.

4. Kumar Suranjit Prasad, Hirnee Patel, Tirtha Patel, Khusbu Patel, Kaliaperumal Selvaraj. Biosynthesis of Se nanoparticles and its effect on UV-induced DNA damage. Colloids Surf B 2013;103:261-6.

5. Prathna TC, Lazar Mathew, N Chandrasekaran, Ashok M Raichur, Amitava Mukherjee. Biomimetic synthesis of nanoparticles: science, technology and applicability. Biomimetics-Learning from nature; 2010. p. 1-20.
6. Siavas Irarani. Green synthesis of metal nanoparticles using plants. Green Chem 2011;13:2638-50.

7. CH Ramamurthy, KS Sampath, P Arun kumar, M Suresh Kumar, V Sujatha, K Prem kumar, et al. Green synthesis and characterization of selenium nanoparticles and its augmented cytotoxicity with doxorubicin on cancer cells. Bioprocess Biosyst Eng 2013;36:1131-9.

8. Mojtaba Shakibaie, Ahmad Reza Shahverdi, Mohammad Ali Faramarzi, Gholam Reza Hassanzadeh, Hamid Reza Rahimi, Omid Sabzevari. Acute and subacute toxicity of novel biogenic selenium nanoparticles in mice. Pharm Biol 2012;51:58-63.

9. Parisa Jafari Fesharaki, Pardis Nazari, Mojtaba Shakibaie, Sassan Rezaie, Maryam Banoee, Mohammad Abdollahi, et al. Biosynthesis of selenium nanoparticles using Klebsiella pneumonia and their recovery by a simple sterilization process. Brazilian J Microbiol 2010;41:461-6.

10. Deepa B, Ganesan V. Biogenic synthesis and characterization of selenium nanoparticles using the flower of bougainvillea spectabilis willd. Int J Sci Res 2013;4:690-5.

11. Kumar Suranjit Prasad, Tapan Amin, Saloni Katuva, Madhu Kumari, Kaliaperumal Selvaraj. Synthesis of water soluble CdS nanoparticles and study of their DNA damage activity. Arabian J Chem 2014. Doi:10.1016/j.arabjc.2014.05.033.

12. Garima Sharma, Ashish Ranjan Sharma, Riju Bhavesh, Jongbong Park, Bilguun Ganbold, Ju-Suk Nam, et al. Biomoleculemediated synthesis of selenium nanoparticles using dried vitis vinifera (Raisin) extract. Molecules 2014;19:2761-70.

13. Dejian Huang, Boxin Ou, Ronald L Prior. The chemistry behind antioxidant capacity assays. J Agric Food Chem 2005;53:1841-56.

14. NS Rajurkar, Kunda Gaikwad. Evaluation of phytochemicals, antioxidant activity and elemental content of adiantum capillus veneris leaves. J Chem Pharm Res 2012;4:365-74.

15. Baby Joseph, S Justin Raj. Pharmacognostic and phytochemical properties of Aloe vera linn-an overview. Int J Pharm Sci Rev Res 2010;4:106-10.

16. Marino B Arnao, Antonio Cano, Manuel Acosta. The hydrophilic and lipophilic contribution to total antioxidant activity. Food Chem 2001;73:239-44.

17. W Brand-Williams, ME Cuvelier, C Berset. Use of free radical method to evaluate antioxidant activity. Lebensm Wiss Technol 1995;28:25-30.

18. Iris FF Benzie, JJ Strain. The ferric reducing ability of plasma (FRAP) as a measure of "antioxidant power": the FRAP assay. Anal Biochem 1996;239:70-6.

19. Deendayal Mandal, Mark E Bolander, Debabrata Mukhopadhyay, Gobinda Sarkar, Priyabrata Mukherjee. The use of microorganisms for the formation of metal nanoparticles and their application. Appl Microbial Biotechnol 2006;69:485-92.

20. Kavita KS, Syed Baker, Rakshith D, Kavitha HU, Yashwantha Rao HC, Harini BP, et al. Plants as green source towards synthesis of nanoparticles. Int Res J Biol Sci 2013;2:66-76. 
21. Maria I Gil, Francisco A, Tomas-Barberan FA, Hess-Pierce B, Kader AA. Antioxidant capacities, phenolic compounds, carotenoids, and vitamin C contents of nectarine, peach, and plum cultivars from California. J Agric Food Chem 2002;50:4976-82.

22. Nidhi Singh, Prasenjit Saha, Karthik Rajkumar, Jayanthi Abraham. Biosynthesis of silver and selenium nanoparticles by Bacillus sp. JAPSK2 and evaluation of antimicrobial activity. Pharm Lett 2014;6:175-81.

23. Shreya Medda, Amita Hajra, Uttiya Deym, Paulomi Bose, Naba Kumar Mondal. Biosynthesis of silver nanoparticles from aloe vera leaf extract and antifungal activity against Rhizopus sp. and Aspergillus sp. Appl Nanosci 2014;5:875-80.

24. Maryam Zahin, Farrukh Aqil, Iqbal Ahmad. The in vitro antioxidant activity and total phenolic content of four Indian medical plants. Int J Pharm Pharm Sci 2009;1:88-95.

\section{How to cite this article}

- Jay Vyas, Shafkat Rana. Antioxidant activity and biogenic synthesis of selenium nanoparticles using the leaf extract of aloe vera. Int J Curr Pharm Res 2017;9(4):147-152. 